\title{
CONSUMO DE TABACO NA MULHER GRÁVIDA: REVISÃO SISTEMÁTICA DA LITERATURA
}

\author{
| Maria André1; Anabela Cândido²; Conceição Santiago³; Olimpia Cruz"; Teresa Carreira ${ }^{5}$; José Amendoeira ${ }^{6} \mid$
}

\section{RESUMO}

CONTEXTO: O tabagismo durante a gravidez é considerado fator de risco quer para a mulher quer para o feto. O Programa Nacional de Prevenção e Controlo do Tabagismo - PNPCT, Direção Geral da Saúde - DGS (2013) contempla nos objetivos operacionais: Caracterizar o consumo de tabaco e a exposição ao fumo ambiental, na mulher grávida, até 2015. Enquanto Escola Promotora de Saúde (EPS) estamos sensíveis a esta temática, e no âmbito da Unidade de Monitorização de Indicadores em Saúde (UMIS) pretendemos continuar a intervir ao nível da comunidade. Esta revisão sistemática da literatura (RSL) surge como forma de conhecer o estado da arte da problemática e de referenciar instrumentos que permitam a caracterização do consumo de tabaco na mulher grávida.

OBJETIVO(S): Identificar instrumentos para a caracterização do consumo de tabaco na mulher grávida.

METODOLOGIA: RSL pelo método PI[C]OD com a questão: Que instrumentos permitem a caracterização do consumo de tabaco na mulher grávida? Com artigos publicados nas bases de dados da interface-EBSCOhost, de 2003-2013. Dos 59 artigos identificados e com a aplicação dos critérios (inclusão/exclusão), resultaram 11 artigos.

RESULTADOS: Da análise dos artigos foram identificados diferentes instrumentos de colheita de dados que permitem a caracterização do consumo de tabaco nas mulheres grávidas, nomeadamente escalas, questionários e testes bioquímicos de avaliação da cotinina.

CONCLUSÕES: Os instrumentos identificados e as estratégias utilizadas para a aplicação dos mesmos dão contributos consistentes para a elaboração de um instrumento de colheita de dados que permita a caracterização do consumo de tabaco na mulher grávida e futura intervenção na comunidade.

PALAVRAS-CHAVE: Mulher grávida; Tabaco; Escalas; Enfermagem

\section{RESUMEN}

\section{"El consumo de tabaco en las mujeres embarazadas: Revisión sistemática de la literatura"}

CONTEXTO: Fumar durante el embarazo es considerado un factor de riesgo, ya sea para la mujer y el feto. El Programa Nacional de Prevención y Control del Consumo de Tabaco - PNPCT Direção Geral da Saúde - DGS (2013) considera en sus objetivos operativos: Caracterizar el consumo de tabaco y la exposición al humo ambiental en las mujeres embarazadas hasta 2015. Mientras que la Escuela Promotora de Salud (EPS) que son sensibles a esta temática, y la Unidade de Monitorização de Indicadores em Saúde (UMIS) tenemos intención de seguir interviniendo a nivel comunitario. Esta revisión sistemática de la literatura (RSL) surge como una forma de conocer el estado del arte en cuanto a caracterización del consumo de tabaco en las mujeres embarazadas.

OBJETIVO(S): Identificar los instrumentos para la caracterización del consumo de tabaco en las mujeres embarazadas.

METODOLOGÍA: RSL método PI[C]OD con la pregunta: ¿Qué instrumentos permiten la caracterización del consumo de tabaco en la mujer embarazada? Con artículos publicados en las bases de datos EBSCOhost, de 2003-2013. De los 59 artículos identificados con aplicación de criterios (inclusión/exclusión), dio como resultado 11 artículos.

RESULTADOS: El análisis de los artículos, se identificaron diferentes instrumentos de recogida de datos que permitan la caracterización del consumo de tabaco en las mujeres embarazadas, incluyendo las escalas, cuestionarios y pruebas bioquímicas de cotinina.

CONCLUSIONES: Las herramientas y estrategias identificadas utilizados para su ejecución dan contribuciones constantes para el desarrollo de un instrumento de recolección de datos que permite la caracterización de fumar en las mujeres embarazadas y futura intervención comunitaria.

\section{DESCRIPTORES: Mujeres embarazadas; Tabaco; Escalas; En- fermería}

\begin{abstract}
"Tobacco consumption in pregnant women: Systematic literature review"

BACKGROUND: Smoking during pregnancy is considered a risk factor for either the woman and the fetus. The National Programme for Prevention and Control of Tobacco Use - PNPCT Direção Geral da Saúde - DGS (2013) considers in their operational objectives: To characterize tobacco use and exposure to environmental smoke in pregnant women until 2015. While Health Promoter School (HPS) we are sensitive to this thematic, and related to the Unidade de Monitorização de Indicadores em Saúde (UMIS) we intend to continue intervening at the community level. This systematic literature review (SLR) emerges as a way of knowing the state of art regarding the problematic as well as referencing tools that allow the characterization of tobacco use in pregnant women.

AIM: Identify instruments for characterization of smoking in pregnant women.

METHODS: SLR by PI[C]OD method with the question: Which instruments allow the characterization of smoking in pregnant woman? With articles published in the databases of the interface-EBSCOhost from 2003 to 2013 . From the 59 articles identified and the application of criteria (inclusion / exclusion), resulted 11 articles.

RESULTS: Analyzing the articles, different data collection instruments that allow the characterization of tobacco use in pregnant women, including scales, questionnaires and biochemical tests of cotinine were identified. CONCLUSIONS: The identified tools and strategies used to implement them give consistent contributions to the development of an instrument of data collection that allows the characterization of smoking in pregnant women and future community intervention.
\end{abstract}

\section{KEYWORDS: Pregnant woman; Tobacco; Scales; Nursing}

Submetido em 30-09-2014

Aceite em 20-01-2015

1 Professora Adjunta no Instituto Politécnico de Santarém, Escola Superior de Saúde de Santarém, Santarém, Portugal, clara.andre@essaude.ipsantarem.pt 2 Professora Adjunta no Instituto Politécnico de Santarém, Escola Superior de Saúde de Santarém, Santarém, Portugal, anabela.candido@essaude.ipsantarem.pt 3 Professora Adjunta no Instituto Politécnico de Santarém, Escola Superior de Saúde de Santarém, Santarém, Portugal, mconceicao.santiago@essaude.ipsantarem.pt 4 Professora Adjunta no Instituto Politécnico de Santarém, Escola Superior de Saúde de Santarém, Santarém, Portugal, olimpia.fonseca@essaude.ipsantarem.pt 5 Professora Adjunta no Instituto Politécnico de Santarém, Escola Superior de Saúde de Santarém, Santarém, Portugal, teresa.carreira@essaude.ipsantarem.pt 6 Investigador integrado na Universidade Católica Portuguesa, Centro Interdisciplinar em Saúde; Professor Coordenador no Instituto Politécnico de Santarém, Escola Superior de Saúde de Santarém, Unidade de Monitorização de Indicadores em Saúde, Unidade de Investigação, 2005-075 Santarém, Portugal, umis.projetos@essaude.ipsantarem.pt

Citação: André, M., Cândido, A., Santiago, C., Cruz, O., Carreira, T., e Amendoeira, J. (2015). Consumo de tabaco na mulher grávida: Revisão sistemática da literatura. Revista Portuguesa de Enfermagem de Saúde Mental (Ed. Esp. 2), 113-118. 


\section{INTRODUÇÃO}

O tabagismo, entendido como um fenómeno social e global, atingindo todas as regiões do mundo e todos os estratos sociais representa, atualmente, o mais importante problema evitável de saúde pública.

Dados epidemiológicos recentes revelam que a nível mundial, morrem por ano cerca de 5,4 milhões de pessoas fumadoras e ex-fumadoras e que a exposição ao fumo ambiental foi responsável pela morte de $46 \%$ de mulheres, $28 \%$ de crianças e $26 \%$ de homens (OMS, 2008; European Comission, 2012, citados por DGS, 2013). Se nos situarmos no nosso país, Portugal apresenta uma das prevalências mais baixas de fumadores e ex-fumadores, comparativamente à média europeia (21\%) (DGS, 2013). Contudo, em relação à intensidade do consumo, apresenta valores próximos da média europeia 14,4 cigarros por dia (DGS, 2013) dado a considerar para a intervenção na comunidade. Assim, perante a tendência crescente e globalização deste fenómeno social, a OMS em 2003, criou a Convenção Quadro, importante instrumento jurídico internacional no domínio da saúde pública, adotado por Portugal, como Estado-Membro da OMS, em 2006 (DGS, 2013).

No contexto do Plano Nacional de Saúde, foi criado, com carater prioritário, o PNPCT perspetivando-se a redução da incidência e prevalência do tabagismo nas diferentes idades do ciclo vital. Este programa está estruturado em função de 3 eixos estratégicos nucleares sendo estes: prevenção da iniciação do consumo, promoção da cessação tabágica e proteção da exposição ao fumo ambiental; e 2 eixos de intervenção transversal, orientados para a informação, educação, avaliação, formação e investigação (DGS, 2013, p.19).

No quadro da implementação de estratégias de prevenção que permitam maximizar o potencial de bem estar materno-fetal, coloca-se o grande desafio de diminuir e/ ou cessar o consumo de tabaco na mulher grávida. Tal como a literatura refere, fumar durante a gravidez ou a exposição continuada a ambientes de fumo (fumador passivo) compromete gravemente a saúde da mãe e do feto. Em particular, a alta prevalência de fumar durante a gravidez, associada a uma diminuição da perfusão placentar contribui para o aumento de abortos espontâneos, prematuridade, restrição de crescimento fetal (Cnattingius, 2004) e aumento das taxas de morbilidade e mortalidade perinatal e infantil (Corbett, 2006).
A Escola Superior de Saúde de Santarém (ESSS) como EPS e, no âmbito da UMIS, com o trabalho que tem vindo a desenvolver na comunidade, valoriza a temática do consumo de tabaco na mulher grávida contribuindo deste modo para a operacionalização do objetivo do PNPCT da DGS (2013): Caracterizar o consumo de tabaco e a exposição ao fumo ambiental, na mulher grávida, até 2015, estando este integrado no eixo estratégico: "Monitorizar, avaliar e promover a formação profissional, a investigação e o conhecimento".

Perante o exposto, surge a necessidade de identificar instrumentos para a caracterização do consumo de tabaco na mulher grávida e propusemo-nos a realizar uma revisão sistemática da literatura.

\section{METODOLOGIA}

Realizámos a RSL tendo por base a estratégia PI[C]OD, formulámos a seguinte pergunta de pesquisa: Que instrumentos permitem a caracterização do consumo de tabaco na mulher grávida? Com o objetivo de identificar instrumentos para a caracterização do consumo de tabaco na mulher grávida.

A pesquisa foi efetuada, com o recurso aos descritores MeSH Browser de pesquisa, após combinação das componentes da estratégia PI[C]OD, na seguinte expressão de pesquisa: "Pregnant Women or Pregnancy or Gestation AND Smoking or Tobacco or "Smoking during pregnancy" AND Epidemiologic methods or Scales or Measures.

Recorremos às bases de dados da Interface - EBSCOhost MEDLINE with full text, CINAHL Plus with full text, Nursing \& AHC: Comp., Cochrane CRCT, MedicLatina, Database of ARE, HTA, Library IS\&TA, Cochrane MR, Cochrane DSR e NHS.

Como limitadores de pesquisa consideramos: Texto completo; Resumo disponível; Analisado por especialista; Prática baseada na evidência; Pelo menos um autor enfermeiro; Friso Cronológico:01/01/2003 a $31 / 07 / 2013$. A pesquisa foi realizada no dia 1 de Agosto de 2013. 
Dando continuidade aos aspetos metodológicos do processo de revisão, e tendo em atenção que cada um dos acrónimos são considerados os elementos fundamentais da questão de pesquisa, possibilitando deste modo a recuperação da melhor informação científica disponível (Higgins \& Green, 2009; Santos, Pimenta e Nobre, 2007), consideramos como critérios de inclusão para os participantes: Estudos que se reportem a mulheres fumadoras durante a gravidez; em relação à intervenção: Caracterização do consumo de tabaco/história do tabagismo das mulheres grávidas; com Comparação ou não; E tendo como resultados: Instrumentos que permitam a caracterização do consumo de tabaco na mulher grávida; e no Desenho todos os tipos de estudo.

Dos 59 artigos identificados após leitura do resumo, do texto completo e da aplicação dos critérios resultaram 11 artigos, sendo 6 quantitativos, 4 mistos e 1 qualitativo, com níveis de evidência entre I e VI (Melnyk \& Fineout-overholt, 2005). Como estratégia de análise utilizamos a análise estruturante (Flick, 2005).

\section{RESULTADOS}

Numa visão global e caracterizadora dos 11 artigos analisados consideramos como estruturante as seguintes dimensões: identificação, participantes, metodologia, intervenções e principais resultados.

Assim, no que diz respeito à Identificação dos estudos considerámos pertinente referenciar o país de origem, ano de publicação e objetivos. A sua maioria, 7, são oriundos dos EUA e os restantes 4 são provenientes do Canadá, Alemanha, Austrália e Equador, quanto ao ano de publicação situa-se entre 2005 e 2012, sendo que a maioria se reporta aos anos 2010 e 2012; em relação aos objetivos constatámos que todos os estudos efetuam uma caracterização do consumo de tabaco durante a gravidez, sendo que 4 deles ainda caracterizam este consumo no período pré-concecional. $\mathrm{O}$ foco de interesse da maioria dos estudos, 8 , centra-se no período pré-natal, 3 remetem-se para os efeitos nocivos no recém-nascido e 1 direciona-se para o risco do reinício dos hábitos tabágicos após a gravidez.

Em relação ao período pré-natal salientamos, ainda, a preocupação em relacionar os hábitos tabágicos com outras variáveis nomeadamente: fatores psicossociais (humor pré-natal, sintomas depressivos e a violência contra a mulher grávida), sociodemográficos (idade gestacional e coabitantes), fatores ambientais (exposição ao fumo ambiental), fatores culturais e consumo de outras substâncias (álcool e substâncias ilícitas).
Quanto às Participantes a maioria dos estudos, 10, reportam-se às mulheres grávidas e 1 a mulheres no pós-parto; quanto à idade, não emerge nenhum grupo etário, estando assim contemplado todo o período fértil; no que se refere à etnia encontram-se participantes afro-americanas, hispânicas, indígenas canadianas e algumas sem etnia definida.

No que se refere às mulheres grávidas, os estudos reportam-se a todos os trimestres da gravidez, no entanto, realçam a primeira consulta pré-natal como o momento de seleção das participantes. Quanto ao estudo das mulheres no pós-parto, constatámos que os dados se reportam ao período da gravidez.

Quanto à Metodologia, e no que se refere ao desenho (D) da pergunta de pesquisa 6 dos estudos utilizam o método quantitativo,4 o tipo misto e 1 o qualitativo.

Em relação às Intervenções todos os estudos utilizam instrumentos que caracterizam o consumo do tabaco durante a gravidez, nomeadamente questionário, escalas e testes bioquímicos de avaliação da cotinina conforme se explícita no Quadro 1. Estes instrumentos são utilizados em diferentes momentos contemplando a fase pré-concecional, ciclo gravídico e pós-parto. Todos os estudos utilizam instrumentos na triagem para caracterização do grupo e ao longo do período de intervenção para caracterização e avaliação dos hábitos tabágicos, relacionando-os com diferentes variáveis; Ressalva-se 1 estudo que utiliza instrumentos para avaliação do impacto das intervenções implementadas. 
Quadro 1 - Artigos que integram a RSL/Instrumentos identificados

\begin{tabular}{|c|c|c|}
\hline No & Artigo & Instrumentos /Referências \\
\hline 2 & $\begin{array}{l}\text { Título: An Integrated Randomized Intervention to Reduce Behavioral and } \\
\text { Psychosocial Risks: Pregnancy and Neonatal Outcomes } \\
\text { Autores; Subramanian, Katz, Rodan, Gantz, El-Khorazaty, Johnson, \& } \\
\text { Joseph. } \\
\text { Ano/País: 2012/EUA }\end{array}$ & Smoke-Free Families - SFF (Melvin, \& Tucker, 2000). \\
\hline 7 & $\begin{array}{l}\text { Título: Consumo y exposición al humo de tabaco en mujeres embarazadas } \\
\text { de Ecuador } \\
\text { Autores; Poletta, López-Camelo, Gili, Montalvo, \& Castilla. } \\
\text { Ano/País: 2010/Equador }\end{array}$ & Questionário dicotómico \\
\hline 16 & $\begin{array}{l}\text { Título: Identifying Women at-Risk for Smoking Resumption after Preg- } \\
\text { nancy } \\
\text { Autores: Merzel, English, \& Moon-Howard. } \\
\text { Ano/País: 2010/EUA }\end{array}$ & $\begin{array}{l}\text { Smoking Assessment Instruments - SAI (English, } \\
\text { Merzel, Moon-Howard, Forthcoming, sd.). } \\
\text { Smoke-Free Families - SFF (Melvin, \& Tucker, 2000; } \\
\text { Melvin, \& Gaffney, 2004). }\end{array}$ \\
\hline 19 & $\begin{array}{l}\text { Título: Independent and Interactive Associations of Prenatal Mood and } \\
\text { Substance Use With Infant Birth Outcomes } \\
\text { Autores: Gyllstrom, Hellerstedt, \& McGovern }\end{array}$ & $\begin{array}{l}\text { Pregnancy Risk Assessment Monitoring System - } \\
\text { PRAMS } \\
\text { http://www.cdc.gov/PRAMS/methodology.htm. }\end{array}$ \\
\hline \multirow[t]{3}{*}{24} & \multirow{3}{*}{$\begin{array}{l}\text { Título:Maternal Prenatal Depressive Symptoms, Nicotine Addiction, and } \\
\text { Smoking-Related Knowledge, Attitudes, Beliefs, and Behaviors } \\
\text { Autores: Orr, Blazer, \& Orr. } \\
\text { Ano/País: 2012/ EUA }\end{array}$} & $\begin{array}{l}\text { Smoke-Free Families - SFF (Melvin, \& Tucker, 2000; } \\
\text { Melvin, \& Gaffney, 2004). }\end{array}$ \\
\hline & & $\begin{array}{l}\text { Teste bioquímico - cotinina (Klebanoff, Levine, Cle- } \\
\text { mens, DerSimonian, \& Wilkins, 1998). }\end{array}$ \\
\hline & & $\begin{array}{l}\text { Questionário - Crenças e conhecimentos sobre taba- } \\
\text { gismo; Fatores ambientais }\end{array}$ \\
\hline 34 & $\begin{array}{l}\text { Título: Prevalence and Correlates of Smoking During Pregnancy: A Com- } \\
\text { parison of Aboriginal and Non-Aboriginal Women in Manitoba } \\
\text { Autores;Heaman, \& Chalmers. } \\
\text { Ano/País:2005/Canadá }\end{array}$ & Questionário estruturado \\
\hline 42 & $\begin{array}{l}\text { Título: Risk Factors for Prenatal Depressive Symptoms Among Hispanic } \\
\text { Women } \\
\text { Autores: Fortner, Pekow, Dole, Markenson \& Chasan-taber } \\
\text { Ano/País:2011/EUA }\end{array}$ & $\begin{array}{l}\text { Pregnancy Risk Assessment Monitoring System - } \\
\text { PRAMS (Williams, Morrow, Lansky, et al. (2003). }\end{array}$ \\
\hline \multirow[t]{3}{*}{44} & \multirow{3}{*}{$\begin{array}{l}\text { Título: Smoking Among Pregnant Women with Medicaid Insurance: Are } \\
\text { Mental Health Factors Related? } \\
\text { Autores; Holtrop, Meghea, Raffo, Biery, Chartkoff, \& Roman. } \\
\text { Ano/País: 2010/EUA - Michigan }\end{array}$} & $\begin{array}{l}\text { Multiple Choice Question - MCQ (Mullen, Carbon- } \\
\text { ari, Tabak, \& Glenday, 1991). }\end{array}$ \\
\hline & & $\begin{array}{l}\text { Pregnancy Risk Assessment Monitoring System - } \\
\text { PRAMS (Tong, Jones, Dietz, D’Angelo, \& Bombard, } \\
\text { 2009). }\end{array}$ \\
\hline & & $\begin{array}{l}\text { Fagerstrom Test for Nicotine Dependence - FTND } \\
\text { (Heatherton, Kozlowski, Frecker, \& Fagerstrom, } \\
\text { 1991). }\end{array}$ \\
\hline 46 & $\begin{array}{l}\text { Título:Smoking During Pregnancy and Postpartum: Smoking Rates and } \\
\text { Intention to Quit Smoking or Resume After Pregnancy } \\
\text { Autores:Hannover, Thyrian, Ebner, Roske, Grempler, Kuhl, Hapke, Fusch, } \\
\text { \& John. } \\
\text { Ano/País: 2008/Alemanha }\end{array}$ & $\begin{array}{l}\text { Fagerström Test for Nicotine Dependence - FTND } \\
\text { (Heatherton, Kozlowski, Frecker, \& Fagerstrom, } \\
\text { 1991). }\end{array}$ \\
\hline 49 & $\begin{array}{l}\text { Título:The association between depresive symptoms and smoking in preg- } \\
\text { nant adolescents } \\
\text { Autores; Bottomleya, \& Lancaster. } \\
\text { Ano/País:2008/Austrália - Melbourne }\end{array}$ & $\begin{array}{l}\text { Smoking Questionnaire - SQ (Lawson's, 1994; Bot- } \\
\text { tomley, \& Lancaster, in preparation). }\end{array}$ \\
\hline 50 & $\begin{array}{l}\text { Título:Use and Smoking in Early Pregnancy and Congenital Cardiac De- } \\
\text { fects } \\
\text { Autores; Mateja, Nelson, Kroelinger, Ruzek, \& Segal. } \\
\text { Ano/País: 2012/EUA }\end{array}$ & $\begin{array}{l}\text { Pregnancy Risk Assessment Monitoring System - } \\
\text { PRAMS (Adams, Shulman Bruce, \& Brogan, 1991; } \\
\text { Colley-Gilbert, Shulman, Fischer, \& Rogers, 1999) }\end{array}$ \\
\hline
\end{tabular}


Como principais resultados verificámos que o consumo de tabaco durante a gravidez é tendencialmente mais frequente quando associado às variáveis sociodemográficas (baixo nível de escolaridade e/ou elevados níveis de escolaridade, baixos níveis socioeconómicos e coabitação com fumadores); demonstram os estudos que existe uma associação entre os sintomas depressivos pré-natais e/ou durante a gravidez e a dependência da nicotina.

As mulheres que fumavam e consumiam álcool apresentavam scores mais elevados de sintomas depressivos. Verificámos, assim, que embora exista um enfase na avaliação de algumas dimensões da saúde mental, os estudos são díspares nas variáveis estudadas, não permitindo obter uma visão clara sobre quais as variáveis que melhor caraterizam a temática em estudo.

No que diz respeito aos efeitos nocivos do consumo do tabaco durante a gravidez para o recém-nascido, os estudos são consistentes com a literatura ao revelarem que os hábitos tabágicos influenciam o baixo peso ao nascer, especialmente durante o terceiro trimestre; por sua vez, um dos estudos mostra que o consumo de tabaco parece melhorar o mau humor da mulher durante a gestação.

Outro dos resultados relevantes é a diminuição do consumo de tabaco após o conhecimento da gravidez, estando este facto associado à crença de que o tabaco prejudica o bebé, no entanto, tendencialmente estas mulheres reiniciam os seus hábitos tabágicos após o parto.

\section{DISCUSSÃO}

Os instrumentos identificados nos diferentes estudos que constituíram esta RSL permitem caracterizar o consumo de tabaco na mulher grávida.

Relativamente aos questionários mobilizados, estes centram-se nos conhecimentos e crenças sobre os efeitos nocivos do consumo de tabaco na mulher grávida e no bebé, no consumo ativo de tabaco na mulher grávida e na exposição ao fumo ambiental do tabaco. A avaliação bioquímica da cotinina foi determinada na saliva e na urina.

No Quadro 2, apresentamos as escalas identificadas e os aspetos mais relevantes que os estudos obtiveram da sua aplicação.
Quadro 2 - Escalas identificadas na caracterização do consumo de tabaco na mulher grávida

\begin{tabular}{|l|l|}
\hline SFF & $\begin{array}{l}\text { Avalia nos fumadores ativos os níveis de dependên- } \\
\text { cia, a cessação tabágica, os momentos de mudança e } \\
\text { as abordagens de intervenção, nos diferentes trimes- } \\
\text { tres da gravidez. }\end{array}$ \\
\hline SAI & $\begin{array}{l}\text { Visa medir a presença de fatores que influenciam a } \\
\text { recaída do consumo de tabaco. Avalia o consumo } \\
\text { de tabaco no período pré-concecional, consumo de } \\
\text { tabaco pelo parceiro, exposição ao fumo ambiental } \\
\text { do tabaco e regras relacionadas com o consumo de } \\
\text { tabaco dentro de casa. }\end{array}$ \\
\hline PRAMS & $\begin{array}{l}\text { Possibilita a caracterização das experiencias e } \\
\text { comportamentos das mulheres durante os períodos } \\
\text { pré-concecional e pré-natal, nomeadamente através } \\
\text { do número de cigarros fumados por dia e a sua } \\
\text { equivalência em número de maços. }\end{array}$ \\
\hline MCQ & $\begin{array}{l}\text { Mede o consumo de tabaco, permitindo às mulheres } \\
\text { identificar o seu hábito de fumar num continuum, } \\
\text { que vai desde fumadoras regulares, redução do } \\
\text { consumo de tabaco durante a gravidez, consumo } \\
\text { ocasional e cessação tabágica durante a gravidez. }\end{array}$ \\
\hline FTND & $\begin{array}{l}\text { Avalia o grau de dependência da nicotina. } \\
\text { tabaculta informações em relação ao consumo de } \\
\text { cional. }\end{array}$ \\
\hline SQ & \begin{tabular}{l} 
Fante a gravidez e no período pré-conce- \\
\hline
\end{tabular} \\
\hline
\end{tabular}

\section{CONCLUSÃO}

A realização desta RSL permitiu-nos conhecer o estado de arte sobre os instrumentos utilizados para a caracterização do consumo de tabaco pela mulher grávida. A identificação dos instrumentos e as estratégias utilizadas para a aplicação dos mesmos suportam o desenvolvimento de instrumentos de colheita de dados, que nos permitam fazer a caracterização futura do consumo de tabaco da mulher grávida no distrito de Santarém.

\section{IMPLICAÇÕES PARA A PRÁTICA CLÍNICA}

O consumo de tabaco durante a gravidez é o mais importante fator de risco modificável em que podemos intervir.

Como EPS consideramos que os resultados desta RSL dão um contributo sustentado para a continuidade do trabalho que estamos a desenvolver, o estudo epidemiológico sobre o consumo de tabaco na mulher grávida, indo ao encontro dos eixos de intervenção transversal, previstos no PNPCT da DGS (2013). 


\section{REFERÊNCIAS BIBLIOGRÁFICAS}

Bottomleya, K. L., \& Lancaster, S. J. (2008). The association between depressive symptoms and smoking in pregnant adolescents. Psychology, Health \& Medicine, 13(5), 574-582.

Cnattingius, S. (2004). The epidemiology of smoking during pregnancy: Smoking prevalence, maternal characteristics, and pregnancy outcomes. Nicotine \& Tobacco Research, 6(Suppl. 2), 125-140. Acedido em Junho, 13 de 2014, em http://ntr.oxfordjournals.org/

Corbett, R. W. (2006). Cuidados de enfermagem durante a gravidez. In D. L. Lowdermilk, \& S. E. Perry, Enfermagem na maternidade (pp. 245-303). Loures: Lusociência.

Direção Geral da Saúde (2013). Programa Nacional para a Prevenção e Controlo do Tabagismo 2012-2016. Lisboa: Direção Geral da Saúde.

Flick, U. (2005). Métodos qualitativos na investigação científica. Lisboa: Monitor.

Fortner, R., Pekow, P., Dole, N., Markenson, G., \& Chasan-Taber, L. (2011). Risk factors for prenatal depressive symptoms among Hispanic women. Maternal \& Child Health Journal, 15(8), 1287-1295.

Gyllstrom, E. M., Hellerstedt, W. L., \& McGovern, P. M. (2011). Independent and Interactive Associations of Prenatal Mood and Substance Use With Infant Birth Outcomes. Maternal \& Child Health Journal, 15(2), 198-204.

Hannover, W., Thyrian, J. R., Ebner, A., Roske, K., Grempler, J., Kuhl, R., Hapke, U., Fusch, C., \& John, U. (2008). Smoking during pregnancy and postpartum: Smoking rates and intention to quit smoking or resume after pregnancy. Journal of Women's Health, 17(4), 631-640.

Heaman, M. I., \& Chalmers, K. (2005). Prevalence and correlates of smoking during pregnancy: A comparison of aboriginal and non-aboriginal women in Manitoba. Birth: Issues in Perinatal Care, 32(4), 299-305.
Higgins, J., \& Green, S. (2009). Cochrane Handbook for Systematic Reviews of Interventions Version 5.0.2. The Cochrane Collaboration, 2009.

Holtrop, J. S., Meghea, C., Raffo, J. E., Biery, L., Chartkoff, S. B., \& Roman, L. A. (2010). Smoking among pregnant women with medicaid insurance: Are mental health factors related?. Maternal \& Child Health Journal, 14(6), 971-977.

Mateja, W. A., Nelson, D. B., Kroelinger, C. D., Ruzek, S., \& Segal, J. (2012). The association between maternal alcohol use and smoking in early pregnancy and congenital cardiac defects. Journal of Women's Health, 21(1), 26-34.

Melnylk, \& Fineout-Overholt (2005). Rating system for hierarchy of evidence.

Merzel, C., English, K., \& Moon-Howard, J. (2010). Identifying women at-risk for smoking resumption after pregnancy. Maternal \& Child Health Journal, 14(4), 600-611.

Orr, S. T., Blazer, D. G., \& Orr, C. (2012). Maternal prenatal depressive symptoms, nicotine addiction, and smoking-related knowledge, attitudes, beliefs, and behaviors. Maternal \& Child Health Journal, 16(5), 973978.

Poletta, F. A., López-Camelo, J. S., Gili, J. A., Montalvo, G., \& Castilla, E.E. (2010). Consumo y exposición al humo de tabaco en mujeres embarazadas de Ecuador. Revista Panamericana de Salud Pública, 27(1), 56-65.

Santos, C. M. C., Pimenta, C. A. M., e Nobre, M. R. C. (2007). A estratégia PICO para a construção da pergunta de pesquisa e busca de evidências. Revista Latinoamericana de Enfermagem, 15(3).

Subramanian, S., Katz, K. S., Rodan, M., Gantz, M. G., El-Khorazaty, N., Johnson, A., \& Joseph, J. (2012). An integrated randomized intervention to reduce behavioral and psychosocial risks: Pregnancy and neonatal outcomes. Maternal \& Child Health Journal, 16(3), 545554. 